\title{
Design study for a kW-class, multi-TW, ps laser
}

\author{
K. Ertel, S. Banerjee, A. Boyle, I. O. Musgrave, W. Shaikh, \\ S. Tomlinson, M. De Vido, T. B. Winstone, \\ A. S. Wyatt, C. B. Edwards, C. Hernandez-Gomez \\ and J. L. Collier
}

\section{Published version information}

Citation: K. Ertel et al. "Design study for a kW-class, multi-TW, ps laser." In: Laser Congress 2017 (ASSL, LAC), OSA Technical Digest online (2017): paper JTu2A.44.

DOI: $\underline{10.1364 / A S S L .2017 . J T U 2 A .44}$

This version is made available in accordance with publisher policies. Please cite only the published version using the reference above.

C2017 Optical Society of America. One print or electronic copy may be made for personal use only. Systematic reproduction and distribution, duplication of any material in this paper for a fee or for commercial purposes, or modifications of the content of this paper are prohibited.

This item was retrieved from ePubs, the Open Access archive of the Science and Technology Facilities Council, UK. Please contact epubs@stfc.ac.uk or go to http://epubs.stfc.ac.uk/ for further information and policies. 


\title{
Design study for a $\mathrm{kW}$-class, multi-TW, ps laser
}

\author{
K. Ertel, S. Banerjee, A. Boyle, I. O. Musgrave, W. Shaikh, S. Tomlinson, M. De Vido, T. B. Winstone, A. S. \\ Wyatt, C. B. Edwards, C. Hernandez-Gomez, J. L. Collier \\ Central Laser Facility, STFC Rutherford Appleton Laboratory, Didcot, OX11 7FZ, United Kingdom \\ klaus.ertel@stfc.ac.uk
}

\begin{abstract}
We explore how the DiPOLE architecture, based on diode-pumped, cryo-cooled $\mathrm{Yb}$ :YAG, could be adapted for direct-CPA ps-pulse generation and conclude that generation of $2 \mathrm{ps}, 70 \mathrm{~J}$ pulses at $10 \mathrm{~Hz}$ repetition rate is feasible.

OCIS codes: (140.3615) Lasers, ytterbium; (140.3480) Lasers, diode-pumped; (140.7090) Ultrafast lasers; (140.3280) Laser amplifiers
\end{abstract}

\section{Introduction}

We have developed a high energy, high repetition rate DPSSL platform called DiPOLE, based on a cryo-cooled, multi-slab Yb:YAG amplifier architecture, that has proven capable of delivering $100 \mathrm{~J}$ pulses of few-ns duration at $10 \mathrm{~Hz}$ repetition rate, making it the first laser, to our knowledge, that has produced such high-energy ns pulses at such high average power [1]. One major application of such a laser is pumping of fs-amplifiers (Ti:sapphire or OPCPA) for the production of multi-Hz PW pulses, which have a multitude of uses, in particular for the realisation of secondary sources, such as laser driven particle, X-ray, or gamma sources.

While Ti:sapphire and OPCPA systems can deliver extremely high peak power pulses, owing to their large bandwidth and resulting short compressed pulse duration, their overall efficiency with regards to average power is rather low, and their overall complexity is large, both as a result of the multiple conversion stages involved.

An alternative is presented by the direct CPA approach, using laser amplifiers which do not require complex laser systems for pumping, but which offer enough bandwidth such that pulse compression to the few-ps to sub-ps range is possible. Such lasers promise increased efficiency, especially if diode pumping is used, and reduced complexity, while the pulse durations and peak powers they offer are still suitable for certain applications [2].

We have therefore started to explore if and how the DiPOLE 100 laser [3] could be converted into a CPA system, with minimum changes to the original set-up in order to preserve the capability to generate arbitrarily shaped ns-pulses of variable length.

A block diagram of the DiPOLE 100 system is shown in Fig 1. The amplifiers that would be used for both CPA and ns operation are: PA1, a regenerative amplifier, based on $\mathrm{Yb}$-doped $\mathrm{CaF}_{2}$, with an output energy of few $\mathrm{mJ}$; PA2, a multi-pass amplifier with Yb:YAG at room temperature as gain medium, producing $100 \mathrm{~mJ}$; MA1, a cryocooled, Yb:YAG multi-pass amplifier producing up to $10 \mathrm{~J}$; and MA2, a scaled-up version of MA1, producing up to $100 \mathrm{~J}$.

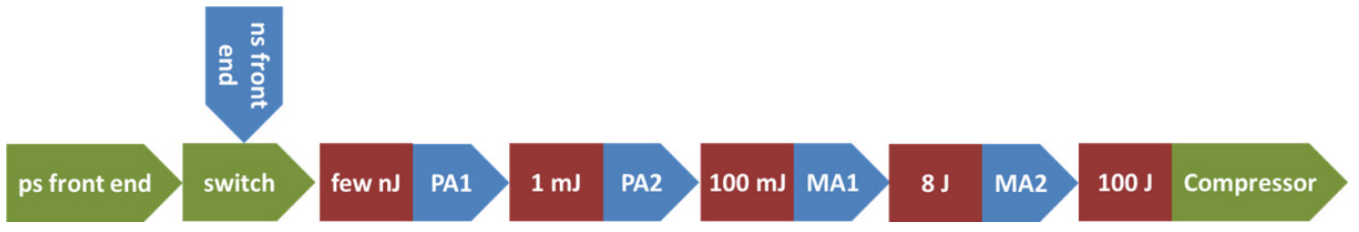

Fig. 1: Main components of DiPOLE 100. Blue: original components of ns laser; green: additional components for CPA operation; red: approximate pulse energy at input of component; PA: pre-amplifier; MA: main amplifier.

In CPA mode, the ns front-end is replaced by a mode-locked oscillator and a stretcher, and a grating compressor is added to the output.

\section{Spectral and gain modelling}

The DiPOLE architecture relies on cryo-cooled main amplifier stages to achieve efficient energy extraction at moderate fluence levels. However, the gain bandwidth of Yb:YAG significantly decreases with temperature, which poses a major challenge for CPA operation. Sufficient spectral width of the amplified pulses is required to achieve a short compressed pulse duration and, even more importantly, to achieve a sufficiently long stretched pulse duration in order to avoid optical damage and detrimental nonlinear effects. 
To simulate amplification of stretched broadband pulses, an existing numerical amplifier model, described in [4], has been adapted to include spectrally resolved emission cross sections, taken from [5]. The model operates in the temporal domain. To convert the temporal pulse shape into a spectral distribution, a stretch factor of $2 \mathrm{~ns} / \mathrm{nm}$ was assumed. The resulting temporal and spectral shapes, obtained after optimizing some parameters, are shown in Fig 2.

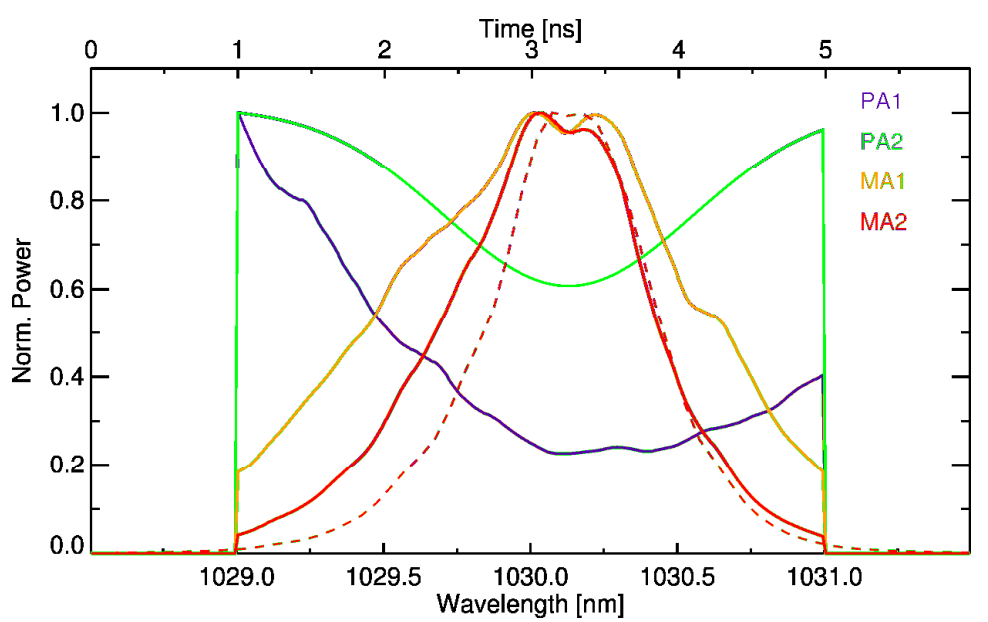

Fig. 2: Simulated pulse/spectral shapes after different amplifier stages. Dashed line: result for a non-optimised input-shape, a $3 \mathrm{~nm}$ wide top-hat.

The calculated pulse energies after the individual stages are $1 \mathrm{~mJ}, 100 \mathrm{~mJ}, 8 \mathrm{~J}$ and $100 \mathrm{~J}$, respectively. The cumulated B-Integral is $1.6 \mathrm{rad}$ and the effective bandwidth (derived from the standard deviation of the spectral distribution and equivalent to the FWHM of a Gaussian curve) is $0.85 \mathrm{~nm}$, corresponding to a transform limited pulse duration of about 2 ps.

The spectra shown in Fig. 2 are the result of some optimisation which includes the following elements:

- Restricting the width of the input spectrum to a range where non-negligible gain is experienced in the last amplifier stage. Spectral components outside this window would unnecessarily extract energy from the earlier stages, while being lost in the latter stages, lowering the overall efficiency. In the scenario presented here the input spectrum was hard-clipped to $2 \mathrm{~nm}$.

- Active shaping of the input spectrum, using a tunable filter in the front-end. A desired shape with a central dip was prescribed for the output of PA2. The required input shape from the front-end was calculated using an iterative algorithm that will be described elsewhere. It is assumed that PA1 does not introduce significant spectral distortion due to the large bandwidth of the $\mathrm{CaF}_{2}$ gain medium.

- Operating the cryo-cooled amplifiers at a higher than usual temperature and compensating for the reduced gain by higher pump energy (within the capabilities of the existing pump sources). The temperatures chosen for PA1 and PA2 were $200 \mathrm{~K}$ and $180 \mathrm{~K}$, respectively, instead of the usual $150 \mathrm{~K}$.

\section{Pulse stretching, compression, and dispersion management}

The results shown in the previous section indicate that a stretch factor of $2 \mathrm{~ns} / \mathrm{nm}$ is required to keep the peak intensity of the stretched pulse within tolerable levels. While novel stretching schemes such as chirped fibre Bragg gratings are being investigated, it is not yet clear whether the required stretch factor and compressibility can be achieved this way. Therefore a conventional, grating-based Offner stretcher has been designed, together with a grating compressor whose dispersion is matched to the stretcher.

Ray-tracing diagrams of stretcher and compressor are shown in Fig. 3. The grating line density is $1740 \mathrm{~mm}^{-1}$ and the incident angle is $56^{\circ}, 11^{\circ}$ away from the Littrow angle. The stretcher has a length of about $5 \mathrm{~m}$ and is a double-pass design where the beam passes the grating a total of eight times. Due to the limited spectral acceptance window of $3 \mathrm{~nm}$, the optics are relatively small, with the grating being the largest component, needing to accommodate a beam footprint of $320 \times 60 \mathrm{~mm}^{2}$.

The compressor is a folded double-pass design with two large gratings with incoming and outgoing beams sitting above each other. The transmission is assumed to be $70 \%$, resulting in $70 \mathrm{~J}$ compressed pulse energy. The footprint of this arrangement is $4.5 \times 2.5 \mathrm{~m}^{2}$. The largest optic in the system is the second grating with a size of $700 \times 460 \mathrm{~mm}^{2}$, resulting in a spectral acceptance window of $2 \mathrm{~nm}$, commensurate with the spectra shown in Fig. 2. The beam size is 
200x200 $\mathrm{mm}^{2}$, yielding a fluence that would be tolerable for gold-coated gratings. However, the high average power, which would result in a significant thermal load and the large off-Littrow angle, that would result in a reduced diffraction efficiency in gold coated gratings, would make the use of dielectric gratings preferable.

Currently though, the safe long-term operating fluence of dielectric gratings, especially under high repetition rate operation, has not yet been established to our knowledge.
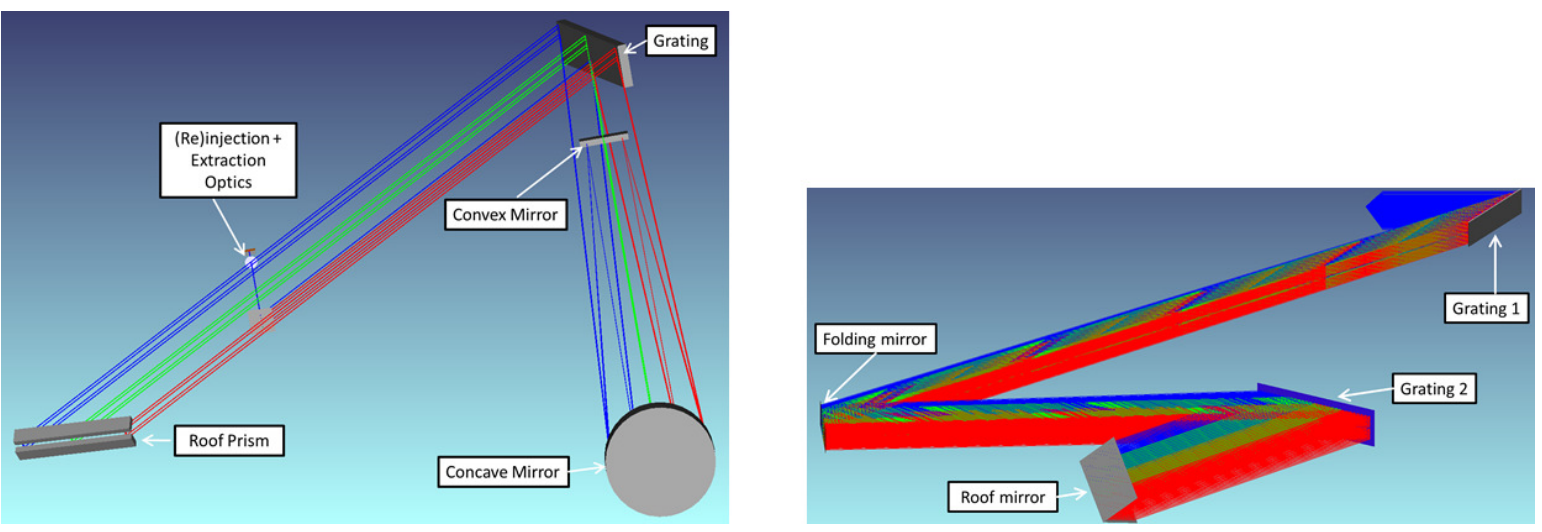

Fig. 3: Ray-tracing models of stretcher (right) and compressor (left).

While grating separation in stretcher and compressor needs to be matched to within in a fraction of a millimetre and grating angles need to be controlled to within few microradians to achieve transform-limited compressed pulse duration, the dispersion of optical components, i.e. bulk dispersion and especially coating dispersion is almost negligible. For example $15 \mathrm{~m}$ of fused silica (in the form of optical fibres in the front-end) would only result in a lengthening of the stretched pulse by about 1 ps and can easily be compensated by a small change in grating distance. Higher order dispersion terms, other than group velocity dispersion, which would require additional degrees of freedom for compensation, are of no concern at all.

\section{Summary}

We have shown that the DiPOLE platform can be adapted to direct CPA operation and has the potential to produce multi-TW pulses at unprecedented pulse energies and average powers. Required for this are careful optimisation of the amplified spectral bandwidth, stretcher and compressor systems with very high dispersion (but limited spectral throughput), and compressor gratings that are not adversely affected by $\mathrm{kW}$ average powers.

\section{References}

[1] P. Mason, M. Divoký, K.Ertel, J. Pilař, T. Butcher, M. Hanuš, S. Banerjee, P. J. Phillips, J. Smith, M. De Vido, A. Lucianetti, C. HernandezGomez, C. Edwards, T. Mocek, and J. Collier, "Kilowatt average power 100 J-level diode pumped solid state laser," Optica 4, 438-439 (2017) .

[2] C. Baumgarten, M. Pedicone, H. Bravo, H. Wang, L. Yin, C. S. Menoni, J. J. Rocca, and B. A. Reagan, "1 J, 0.5 kHz repetition rate picosecond laser," Opt. Lett. 41, 3339-3342 (2016).

[3] S. Banerjee, P. D. Mason, K. Ertel, P. J. Phillips, M. De Vido, O. Chekhlov, M. Divoky, J. Pilar, J. Smith, T. Butcher, A. Lintern, S. Tomlinson, W. Shaikh, C. Hooker, A. Lucianetti, C. Hernandez-Gomez, T. Mocek, C. Edwards, and J. L. Collier, "100 J-level nanosecond pulsed diode pumped solid state laser," Opt. Lett. 41, 2089-2092 (2016) .

[4] K. Ertel, S. Banerjee, P. D. Mason, P. J. Phillips, M. Siebold, C. Hernandez-Gomez, and J. C. Collier, "Optimising the efficiency of pulsed diode pumped Yb:YAG laser amplifiers for ns pulse generation.," Opt. Express 19, 26610-26626 (2011).

[5] J. Koerner, J. Hein, M. Kahle, H. Liebetrau, M. Lenski, M. Kaluza, M. Loeser, and M. Siebold, "Temperature dependent measurement of absorption and emission cross sections for various Yb3+ doped laser materials," Proc. SPIE 8080, 808003 (2011) 